\title{
Single-Dose Oritavancin Compared to Standard of Care IV Antibiotics for Acute Bacterial Skin and Skin Structure Infection in the Outpatient Setting: A Retrospective Real-World Study
}

\author{
Patrick J. Anastasio · Pete Wolthoff · Annmarie Galli · Weihong Fan
}

Received: November 21, 2016 / Published online: January 11, 2017

(C) The Author(s) 2017. This article is published with open access at Springerlink.com

\begin{abstract}
Introduction: Cost-containment strategies are shifting the treatment of acute bacterial skin and skin structure infections (ABSSSI) from inpatient to outpatient settings. Current standard of care (SoC) requires multiple-dose regimens, which are associated with high hospitalization rates and high costs. Oritavancin, a new single-dose antibiotic for ABSSSI, may be suitable for outpatient therapy. This analysis evaluates the effectiveness, costs, and resource utilization of oritavancin vs. SoC in a real-world, outpatient setting.
\end{abstract}

Enhanced content To view enhanced content for this article go to http://www.medengine.com/Redeem/ D257F060381D5215.

P. J. Anastasio

Emerald Coast Infectious Disease Medical Group, 917 Mar Walt Drive, Fort Walton Beach, FL 32547, USA

P. Wolthoff

Medical Data Analytics, 5 Sylvan Way, Parsippany, NJ 07054, USA

A. Galli

Research Query, 33 Glen Green, Winchester, MA 01890, USA

W. Fan $(\bowtie)$

The Medicines Company, 8 Sylvan Way,

Parsippany, NJ 07054, USA

e-mail: weihong.fan@THEMEDCO.com
Methods: A single-site, retrospective chart review was conducted of 118 adult patients diagnosed with ABSSSI and treated with either single-dose oritavancin or multi-dose SoC therapy between 6 August 2014 and 30 June 2015. Patients were assigned to two matched cohorts: oritavancin and SoC. Primary clinical effectiveness endpoints was the success (cured or improved) at 5-30 days after the course of antibiotic therapy has been completed. Secondary economic endpoints were total costs and healthcare resource utilization.

Results: Oritavancin showed comparable clinical effectiveness vs. multi-dose SoC in the outpatient setting. A similar percentage of patients in the oritavancin $(90.2 \%)$ and SoC cohorts $(77.4 \%)$ achieved successful outcomes ("cure" or "improved"), with the cure rate higher for oritavancin $(73.2 \%)$ vs. SoC (48.4\%; $P=0.0315)$. Oritavancin's clinical effectiveness was consistent across patient subgroups with varying demographic, clinical, and ABSSSI characteristics. Oritavancin was consistently associated with lower costs (per-patient savings \$2319) and reduced resource utilization measures, and it required just 1.0 day of therapy vs. 7.2 days for SoC.

Conclusion: Oritavancin is well suited for the outpatient treatment of ABSSSI. Compared with SoC, oritavancin offers comparable effectiveness, is more economical, and requires fewer healthcare resources. 
Keywords: Anti-bacterial agents therapy; Intravenous infusions; Oritavancin; Outpatient infusion therapy; Staphylococcal skin infections

\section{INTRODUCTION}

Total US healthcare spending now exceeds \$3 trillion annually, or approximately $\$ 9523$ per person, and $32 \%$ of that cost is spent on hospital care [1]. Acute bacterial skin and skin structure infections (ABSSSI) represent the tenth leading cause for non-maternal/non-neonatal hospital admissions, comprising a total of 569,259 admissions in 2013 and representing $\$ 14$ billion in healthcare spending for hospital care alone [2]. Despite being a top-ten reason for admission, the mortality rate for these infections is only $0.08 \%$ [3].

The high utilization of the hospital inpatient setting for a low acuity condition is due in part to the increasing incidence of community-acquired methicillin-resistant Staphylococcus aureus (CA-MRSA), which comprises up to $60 \%$ of all ABSSSI cases [4-6]. Treatment of MRSA frequently requires intravenous (IV) antibiotics, and because of the time it takes to identify the pathogen, patients are often admitted and treated empirically with IV MRSA-active antibiotics when MRSA is suspected.

Infectious Disease Society of America (IDSA) guidelines indicate that treatment for severe ABSSSI should include antibiotic treatment with IV antibiotics including vancomycin, daptomycin, linezolid, telavancin, or ceftaroline, all of which require multiple doses over several days [7]. Further, vancomycin requires regular serum monitoring for toxicity, another reason for potential hospital admission. A 2015 study funded by the US Centers for Disease Control and Prevention found that emergency room (ER) physicians cited the need for multi-dose intravenous (IV) antibiotics as a reason for admission for $85 \%$ of ABSSSI admissions, with IV antibiotics the sole reason for admission in $41.5 \%$ of cases [8]. Similarly, a 2015 study determined that $67 \%$ of patients hospitalized for ABSSSI had no life-threatening conditions and one or no comorbidities, indicating that a substantial portion of hospitalized ABSSSI patients have no clinical rationale for admission [3].

The cost-saving potential of treating ABSSSI patients in the outpatient instead of inpatient setting may be substantial. Non-drug medical costs for ABSSSI care has been estimated to range from $\$ 9813$ to $\$ 18,014$ in the inpatient setting compared with $\$ 4039$ to $\$ 4924$ for outpatient management [5]. Clinical studies indicate that outcomes may be equally good in the outpatient setting [3, 8], although delivery of successful outpatient care may be difficult for both provider and patient when multiple days of IV therapy are required.

Orbactiv $^{\circledR}$ (oritavancin for injection, The Medicines Company, Parsippany, NJ), a novel semisynthetic lipoglycopeptide antibiotic, is approved to treat ABSSSI caused by gram-positive pathogens, including MRSA. Oritavancin is administered as a one-time, single-dose infusion, making it a strong candidate for outpatient treatment of ABSSSI. The introduction of newer antibiotics like oritavancin, with one-time dosing and fewer intensive monitoring requirements, may be a good solution to the current pressure to manage healthcare costs through reduction of hospital utilization while maintaining quality of care [9-11]. However, little is known about the outcomes and costs of ABSSSI treatment in a real-world outpatient setting. The objective of this analysis was to compare and evaluate clinical outcomes, healthcare costs, and resource utilization associated with single-dose oritavancin vs. multi-dose standard of care (SoC) antibiotics for the treatment of ABSSSI in a real-world outpatient setting.

\section{METHODS}

\section{Study Design}

This single-center, retrospective, observational study evaluated patients treated for ABSSSI caused by suspected or confirmed gram-positive pathogens with single-dose oritavancin or SoC antibiotics in an outpatient infusion center. Patient charts were abstracted 
retrospectively to determine study eligibility. Demographic and infection characteristics, microbiological laboratory results, clinical outcomes, and resource utilization data were extracted from patient records. Patients who received at least one $1200 \mathrm{mg}$ dose of oritavancin between 6 August 2014 and 30 June 2015 were matched to patients who received SoC antibiotics (defined by protocol as any IV antibiotics indicated for ABSSSI and routinely used in practice at this infusion center). Both cohorts were evaluated during the index treatment period, which started when the patient received the first dose of study drug at the infusion center and ended when the patient completed the course of study drug, and for 30 days after index treatment, to assess outcomes, related resource use, and costs.

\section{Setting}

The study took place at Emerald Coast Infectious Diseases Medical Group, PA, a single-center, stand-alone, outpatient infusion center located in Fort Walton Beach, Florida, USA.

\section{Patients}

Patients who met the following criteria were eligible for study enrollment: age $\geq 18$ years with a confirmed diagnosis of wound infection, cellulitis/erysipelas, or major cutaneous abscess suspected/proven to be due to gram-positive pathogens, including MRSA, between 6 August 2014 and 30 June 2015, who received either single-dose oritavancin or SoC antibiotics. It was required that all medical chart data be available for abstraction into a case report form. Patients were excluded from the study if they were enrolled in other ABSSSI-related clinical trial(s) between 6 August 2014 and 30 June 2015. Lesion size measurements were not routinely collected in patient charts and were therefore not used as eligibility criteria. All patients met the criteria for ABSSSI diagnosis according to the investigating physician.
Patients with a confirmed ABSSSI diagnosis who received oritavancin at the outpatient infusion center were enrolled first and then matched on a 1:1 basis with patients with a confirmed ABSSSI diagnosis who received SoC antibiotics. Three mandatory matching criteria were applied: age ( $<65$ or $\geq 65$ years), history of diabetes (type 1 or type 2 , or none), and infection type (cellulitis, wound, or abscess). If an oritavancin-treated patient could be matched to more than one SoC-treated patient based on these mandatory criteria, four additional matching criteria were then applied from highest to lowest priority: body mass index (BMI $<30$ or $\geq 30 \mathrm{~kg} / \mathrm{m}^{2}$ ), insurance status (Medicare, Medicaid, commercial, or self-pay), gender (male or female), and fever $\left(<100.4\right.$ or $\left.\geq 100.4^{\circ} \mathrm{F}\right)$.

Patient baseline and post-treatment vital sign data were collected, including blood pressure, temperature, and heart and respiratory rate. When available, baseline microbiology was assessed to determine whether infections comprised gram-positive organisms only or both gram-negative and -positive organisms. Patients who presented with Staphylococcus aureus organisms were further delineated into methicillin-resistant Staphylococcus aureus (MRSA) or methicillin-susceptible Staphylococcus aureus (MSSA).

Prior to study initiation, a pilot study was conducted at the infusion center site to confirm the availability of comprehensive medical records data for both study cohorts and to identify any data access issues that might arise. Fifty-nine oritavancin patients were found eligible for inclusion; a total of 200 SoC patients were identified as the eligible matching pool.

\section{Primary Effectiveness Outcome}

Clinical response was classified as "cured," "improved," "failure," or "non-evaluable" and assessed at 5-30 days after the course of antibiotic therapy had been completed in the outpatient center. This approach followed standard practice at this center, in which 
patients are scheduled for a follow-up visit within 30 days of treatment whenever possible. The clinical definitions were as follows: (a) cured: resolved clinical signs and symptoms alongside clinician documentation of cure and/or no additional antibiotic therapy prescribed; (b) improved: partial resolution of clinical signs and symptoms, clinician documentation of improvement and/or no additional antibiotic therapy needed; (c) failure: inadequate resolution or new or worsening clinical signs and symptoms, such that additional antibiotic therapy is necessary for treatment of the infection; (d) non-evaluable: inability to determine response because the patient record did not contain the necessary information.

Following this, the percentage of evaluable patients classified as "success" (defined as cured or improved) was calculated and compared between the oritavancin and SoC cohorts.

\section{Economic Outcomes}

Total healthcare costs were defined as the costs incurred at the infusion center from study drug initiation (oritavancin or SoC antibiotic) to the end of the study period. Total costs were further broken down into: medication costs, drug administration costs, IV supply costs, office/ outpatient visit costs, ABSSSI procedure costs, hospital care costs, and other. The infusion center billing charges were collected. Medication costs were estimated based on wholesale acquisition cost and drug dosage; all non-medication services costs were estimated based on billing charges and a unified cost-to-charge ratio (CCR) of 1:2. The CCR of 1:2 was estimated based on ratios of Centers for Medicare and Medicaid Services physician fee payments in the Florida area vs. actual charges.

In addition, healthcare resource utilization during the index treatment period and for 30 days before and after the index treatment period was evaluated for both groups. Healthcare resource utilization metrics included: prior ER visits or hospitalization (including for the same episode of skin infection within 30 days of first dose of index antibiotic); additional antibiotics needed during the index and post-index periods; number of days for infusion center visits; additional clinical follow-up such as ER visits, hospitalization or re-hospitalization for the original episode of care (within 30 days of the last dose of index antibiotic therapy at the infusion center).

\section{Statistical Analysis}

All data were summarized by treatment group (oritavancin, SoC) using descriptive statistics and graphs. Due to the descriptive nature of the study, no formal sample size calculations were conducted. Descriptive statistics for continuous variables included the mean, standard deviations (SD), medians, and minimum and maximum. Categorical variables were summarized by frequencies and percentages. Comparisons between the oritavancin and SoC groups were conducted using chi-square or Fisher exact tests (cell count $<5$ ) for categorical and Wilcoxon rank-sum tests for continuous variables. For the primary effectiveness outcome, the success rate was defined as:

Success rate $(\%)$

$$
\begin{aligned}
& =\frac{\text { Number of patients with cure or improved }}{\text { Number of patients with cure, improved, or failure }} \\
& \times 100 \% \text {. }
\end{aligned}
$$

The success rate difference between oritavancin and SoC groups (=rate of SoC group - rate of oritavancin) was calculated, and the 95\% two-sided confidence interval (CI) of this observed difference was constructed. $P$ values were calculated based on the chi-square test. Furthermore, the success rate was analyzed by various clinically relevant subgroups, such as age, gender, diabetes status, infection type, and others. No alpha adjustment was made for multiple comparisons. For the economic outcome, total healthcare costs were summarized as mean (SD) and median (minimum, maximum) by oritavancin and SoC groups and compared using Wilcoxon rank-sum tests. Subgroup analyses were conducted for both clinical and economic outcomes. With the exception of a sensitivity 
analysis on the primary effectiveness outcome (conducted the explore the impact of missing data on results), missing data values or unknown data were not imputed. Data processing, summarization, and analyses were performed using SAS version 9.2 (SAS Inc., Cary, NC, USA).

\section{Sensitivity Analysis}

A sensitivity analysis was performed to evaluate the robustness of the primary effectiveness outcome of success rate. In the primary analysis of clinical success, patients with non-evaluable data were excluded entirely because no information was available. Sensitivity analyses were conducted by creating two scenarios using the non-evaluable data. First, non-evaluable responses were categorized as "cured or improved" and included in both the denominator and numerator when calculating the success rate. Second, non-evaluable responses were re-categorized as "failure" and included in denominator but not in the numerator. All other effectiveness comparisons were conducted in this fashion.

\section{Compliance with Ethics Guidelines}

All procedures followed were in accordance with ethical standards. A waiver of institutional review consent was granted by the New England Internal Review Board (NEIRB). This study was conducted retrospectively, and all protected, patient-identifiable information was not shared with the Sponsor or anyone outside of the study site. Data abstraction, de-identification, and entry were performed onsite at the infusion clinic by an independent Clinical Research Organization, Medical Data Analytics (MDA) of Parsippany, NJ.

All procedures followed were in accordance with the ethical standards of the responsible committee on human experimentation (institutional and national) and with the Helsinki Declaration of 1964, as revised in
2013. This article is based on previously completed treatment of patients and does not involve any new interventions performed on human or animal subjects by any of the authors. This was a retrospective study; informed consent was waived.

\section{RESULTS}

\section{Patient Characteristics}

A total of 118 patients (59 oritavancin and 59 SoC) met all mandatory matching criteria and were included in the study. As shown in Table 1, patients were similar in terms of mean age (65.3 and 63.8 years in oritavancin and SoC, respectively), primarily female $(n=30$ and $n=38)$, and predominantly white $(n=55$ and $n=53)$. SoC patients received the following IV antibiotics: daptomycin $(n=34,58 \%)$, dalbavancin $(n=15,25 \%)$, vancomycin $(n=3$, $5 \%)$, or other antibiotics $(n=7,12 \%)$. Dosing schedule of drugs was based on prescribing information: daptomycin once daily, dalbavancin once weekly for 2 weeks; and vancomycin twice daily. Choice of antibiotic was based on physician discretion considering the best option for each individual patient. All treatment decisions were made prior to the initiation of this study.

Also shown in Table 1, 93.2\% $(n=110)$ of all patients presented with at least one comorbidity, the most common being hypertension ( $n=74,62.7 \%)$, hyperlipidemia $(n=53,44.9 \%)$, and diabetes $(n=36,30.5 \%)$. The leg was the most common infection site $(n=48,40.7 \%)$, followed by the foot $(n=20$, $16.9 \%)$ and arm $(n=15,12.7 \%)$. Most patients were afebrile, with only $3.4 \% \quad(n=2)$ of oritavancin and $1.7 \%(n=1)$ of SoC patients presenting with fever $\left(\geq 100.4^{\circ} \mathrm{F}\right)$.

Baseline microbiology data (Table 2) were available for $23.7 \%(n=14)$ of oritavancin and $20.3 \%(n=12)$ of SoC patients. All patients with microbiology data presented with at least one gram-positive pathogen. Four patients (2 oritavancin and 2 SoC) had a mix of gram-positive and -negative pathogens. Staphylococcus aureus was the most common 
Table 1 Patient demographics and baseline characteristics

\begin{tabular}{|c|c|c|c|c|}
\hline & Oritavancin $(N=59)$ & SoC $(N=59)$ & Total $(N=118)$ & $P$ value \\
\hline \multicolumn{5}{|l|}{ Age (years) } \\
\hline Mean (SD) & $65.3(16.7)$ & $63.8(16.5)$ & $64.6(16.5)$ & \\
\hline Median (min, max) & $68(23,94)$ & $68(18,90)$ & $68(18,94)$ & 0.572 \\
\hline$\geq 65$ years, $n(\%)$ & $34(57.6 \%)$ & $34(57.6 \%)$ & $68(57.6 \%)$ & 1.000 \\
\hline Male, $n(\%)$ & $29(49.2 \%)$ & $21(35.6 \%)$ & $50(42.4 \%)$ & 0.136 \\
\hline Race, $n(\%)$ & & & & 0.878 \\
\hline White & $55(93.2 \%)$ & $53(89.8 \%)$ & $108(91.5 \%)$ & \\
\hline Black & $3(5.1 \%)$ & $3(5.1 \%)$ & $6(5.1 \%)$ & \\
\hline Asian & $0(0.0 \%)$ & $1(1.7 \%)$ & $1(0.8 \%)$ & \\
\hline Unknown & $1(1.7 \%)$ & $2(3.4 \%)$ & $3(2.5 \%)$ & \\
\hline \multicolumn{5}{|l|}{ Weight (kg) } \\
\hline Mean (SD) & $84.4(27.0)$ & $88.6(31.9)$ & $86.5(29.5)$ & \\
\hline Median $(\min , \max )$ & $81.6(45,205)$ & $79.8(39,188)$ & $80.3(39,205)$ & 0.698 \\
\hline \multicolumn{5}{|l|}{ Height $(\mathrm{cm})$} \\
\hline Mean (SD) & $170.3(9.2)$ & $170.4(11.4)$ & $170.3(10.3)$ & \\
\hline Median $(\min , \max )$ & $170.2(152,188)$ & $167.6(150,196)$ & $170.2(150,196)$ & 0.802 \\
\hline \multicolumn{5}{|l|}{ BMI $\left(\mathrm{kg} / \mathrm{m}^{2}\right)$} \\
\hline Mean (SD) & $28.9(8.6)$ & $30.2(9.4)$ & $29.6(9.0)$ & \\
\hline Median $(\min , \max )$ & $28.0(17.2,72.9)$ & $27.1(16.6,61.3)$ & $27.6(16.6,72.9)$ & 0.714 \\
\hline$\geq 30 \mathrm{~kg} / \mathrm{m}^{2}, n(\%)$ & $19(32.2 \%)$ & $22(37.3 \%)$ & $41(34.7 \%)$ & 0.562 \\
\hline Insurance status, $n(\%)$ & & & & 0.352 \\
\hline Medicare & $37(62.7 \%)$ & $31(52.5 \%)$ & $68(57.6 \%)$ & \\
\hline Medicaid & $0(0.0 \%)$ & $1(1.7 \%)$ & $1(0.8 \%)$ & \\
\hline Commercial & $22(37.3 \%)$ & $27(35.8 \%)$ & $49(41.5 \%)$ & \\
\hline Patient referral location, $n(\%)$ & & & & 0.478 \\
\hline ER & $4(6.8 \%)$ & $1(1.7 \%)$ & $5(4.2 \%)$ & \\
\hline Hospital, observational unit & $1(1.7 \%)$ & $0(0.0 \%)$ & $1(0.8 \%)$ & \\
\hline Hospital, inpatient & $13(22.0 \%)$ & $15(25.4 \%)$ & $28(23.7 \%)$ & \\
\hline Physician's office & $41(69.5 \%)$ & $41(69.5 \%)$ & $82(69.5 \%)$ & \\
\hline $\mathrm{LTCF} / \mathrm{SNF}$ & $0(0.0 \%)$ & $1(1.7 \%)$ & $1(0.8 \%)$ & \\
\hline Unknown & $0(0.0 \%)$ & $1(1.7 \%)$ & $1(0.8 \%)$ & \\
\hline Patients with any comorbidity, $n(\%)$ & $53(89.8 \%)$ & $57(96.6 \%)$ & $110(93.2 \%)$ & 0.272 \\
\hline Most common comorbidities, $n(\%)$ & & & & \\
\hline
\end{tabular}


Table 1 continued

\begin{tabular}{|c|c|c|c|c|}
\hline & Oritavancin $(N=59)$ & SoC $(N=59)$ & Total $(N=118)$ & $P$ value \\
\hline Diabetes & $18(30.5 \%)$ & $18(30.5 \%)$ & $36(30.5 \%)$ & 1.000 \\
\hline Chronic obstructive pulmonary disease & $8(13.6 \%)$ & $5(8.5 \%)$ & $13(11.0 \%)$ & 0.378 \\
\hline Hypertension & $35(59.3 \%)$ & $39(66.1 \%)$ & $74(62.7 \%)$ & 0.446 \\
\hline Hyperlipidemia & $25(42.4 \%)$ & $28(47.5 \%)$ & $53(44.9 \%)$ & 0.579 \\
\hline Depression & $10(16.9 \%)$ & $12(20.3 \%)$ & $22(18.6 \%)$ & 0.636 \\
\hline Coronary artery disease & $14(23.7 \%)$ & $16(27.1 \%)$ & $30(25.4 \%)$ & 0.672 \\
\hline Renal disease & $11(18.6 \%)$ & $8(13.6 \%)$ & $19(16.1 \%)$ & 0.452 \\
\hline Disease state/condition, $n(\%)$ & & & & 1.000 \\
\hline Cellulitis/erysipelas & $34(57.6 \%)$ & $34(57.6 \%)$ & $68(57.6 \%)$ & \\
\hline Major cutaneous abscess & $14(23.7 \%)$ & $14(23.7 \%)$ & $28(23.7 \%)$ & \\
\hline Wound infection & $11(18.6 \%)$ & $11(18.6 \%)$ & $22(18.6 \%)$ & \\
\hline Traumatic & $6(10.2 \%)$ & $3(5.1 \%)$ & $9(7.6 \%)$ & \\
\hline Surgical & $5(8.5 \%)$ & $8(13.6 \%)$ & $13(11.0 \%)$ & \\
\hline Baseline temperature $\geq 100.4^{\circ} \mathrm{F}, n(\%)$ & $2(3.4 \%)$ & $1(1.7 \%)$ & $3(2.5 \%)$ & \\
\hline \multicolumn{5}{|l|}{ Body part/location of infection, $n(\%)$} \\
\hline Face & $2(3.4 \%)$ & $3(5.1 \%)$ & $5(4.2 \%)$ & \\
\hline Neck & $0(0.0 \%)$ & $1(1.7 \%)$ & $1(0.8 \%)$ & \\
\hline Chest & $1(1.7 \%)$ & $1(1.7 \%)$ & $2(1.7 \%)$ & \\
\hline Trunk & $6(10.2 \%)$ & $7(11.9 \%)$ & $13(11.0 \%)$ & \\
\hline Back & $0(0.0 \%)$ & $3(5.1 \%)$ & $3(2.5 \%)$ & \\
\hline Arm & $10(16.9 \%)$ & $5(8.5 \%)$ & $15(12.7 \%)$ & \\
\hline Hand & $6(10.2 \%)$ & $6(10.2 \%)$ & $12(10.2 \%)$ & \\
\hline Buttock & $3(5.1 \%)$ & $4(6.8 \%)$ & $7(5.9 \%)$ & \\
\hline Leg & $27(45.8 \%)$ & $21(35.6 \%)$ & $48(40.7 \%)$ & \\
\hline Foot & $10(16.9 \%)$ & 10 (16.9\%) & $20(16.9 \%)$ & \\
\hline Groin & $1(1.7 \%)$ & $0(0.0 \%)$ & $1(0.8 \%)$ & \\
\hline
\end{tabular}

$B M I$ body mass index, ER emergency room, $L T C F$ long-term care facility, $S D$ standard deviation, $S N F$ skilled nursing facility, $S o C$ standard of care

causative pathogen identified in both groups $(14 / 14$ in oritavancin and 9/12 in SoC). In $S$. aureus isolates, MRSA was found in $78.6 \%$ of oritavancin patients and $77.8 \%$ of SoC patients, consistent with recent MRSA epidemiological findings [12].

\section{Clinical Effectiveness Results}

Clinical effectiveness was assessed at a prescheduled follow-up visit, which took place 5-30 days after completion of index treatment. 
Table 2 Baseline infection-site culture

\begin{tabular}{llll}
\hline & Oritavancin $(N=59)$ & SoC $(N=59)$ & Total $(\boldsymbol{N}=\mathbf{1 1 8})$ \\
\hline Patients with microbiology results, $n(\%)$ & $14(23.7 \%)$ & $12(20.3 \%)$ & $26(22.0 \%)$ \\
Gram-positive organisms only & $12(20.3 \%)$ & $10(16.9 \%)$ & $22(18.6 \%)$ \\
Both gram-positive and -negative organisms & $2(3.4 \%)$ & $2(3.4 \%)$ & $4(3.4 \%)$ \\
Gram-negative organisms only & $0(0.0 \%)$ & $0(0.0 \%)$ & $0(0.0 \%)$ \\
Patients with gram-positive organisms, $n$ & 14 & 12 & 26 \\
Staphylococcus aureus, $n$ & 14 & 9 & 23 \\
MRSA, $n$ & 11 & 7 & 18 \\
MSSA, $n$ & 3 & 2 & 5 \\
Staphylococcus lugdunensis, $n$ & 0 & 2 & 2 \\
Enerococcus faecalis, $n$ & 2 & 3 & 5 \\
\hline
\end{tabular}

MRSA methicillin-resistant Staphylococcus aureus, MSSA methicillin-sensitive Staphylococcus aureus, SoC standard of care

Mean days to follow-up was 16.7 for oritavancin and 17.6 for SoC. Similar clinical effectiveness of success rate at 5-30 days post-treatment was observed between oritavancin and SoC antibiotics $(90.2 \%$ vs. $77.4 \%, 95 \%$ CI of difference: $-4.5 \%, \quad 30.1 \%, \quad P=0.134)$ and across all subgroups (Fig. 1). The clinical cure rate was statistically higher in oritavancin patients (73.2\%) vs. SoC patients (48.4\%; $P=0.0315)$. Within the SoC group, rates of success and cure for specific IV antibiotics were: daptomycin $68.8 \%$ success and $50.0 \%$ cure; dalbavancin $75.0 \%$ success and $37.5 \%$ cure; vancomycin $100.0 \%$ success and $33.3 \%$ cure.

\section{Sensitivity Analysis of Clinical Effectiveness Results}

Sensitivity analyses of clinical effectiveness responses (cured, improved, failure, or non-evaluable) found that results remained similar regardless of how non-evaluable patients were accounted. When non-evaluable patients were categorized as treatment failures, the clinical success percentage remained higher in the oritavancin than SoC cohort (oritavancin, 62.7\%; SoC, 40.7\%; $P=0.0166$ ). Likewise, when non-evaluable patients were treated as successes, the percentage of patients with clinical success was similar across both cohorts (oritavancin, 93.2\%; SoC, 88.1\%; $P=0.3422$ ).

\section{Economic Results}

Mean total costs were lower for oritavancin compared with SoC antibiotics (\$4035 vs. \$6354, $P=0.0107)$, representing $\$ 2319$ per patient savings with oritavancin (Fig. 2). This cost differential was driven by both lower medication costs for oritavancin compared with SoC antibiotics ( $\$ 3260$ vs. $\$ 5157, P=0.0038$ ) and reduced non-medication related costs ( $\$ 830$ vs. $\$ 1198, P<0.0001)$, such as infusion supplies ( $\$ 19$ vs. \$84), infusion administration personnel (\$149 vs. $\$ 722$ ), and outpatient/office costs (\$203 vs. \$276). Costs during the index treatment period alone were also lower for oritavancin compared with SoC antibiotics (\$3114 vs.. \$5144, $P=0.0009$ ). Costs during the 30 -day post index treatment period were comparable for both cohorts ( $\$ 921$ vs. $\$ 1210$, NS).

Mean total costs for oritavancin patients during the study period were predictable and consistent across all clinical subgroups (Fig. 3), ranging from $\$ 3600$ to $\$ 4200$. Total costs for SoC subgroups varied substantially, from $\$ 4400$ to $\$ 9700$. Savings with oritavancin were 


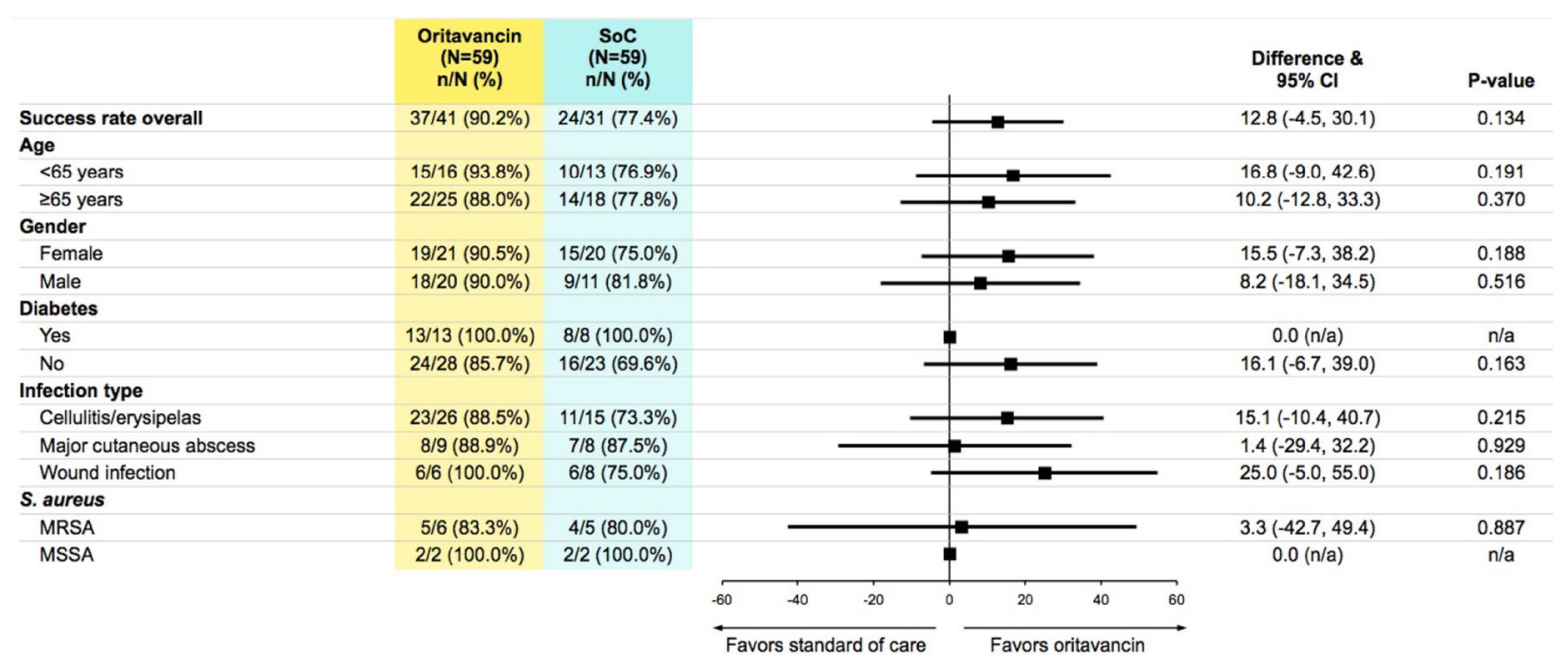

Fig. 1 Clinical response evaluation at 5-30 days post-treatment by subgroup

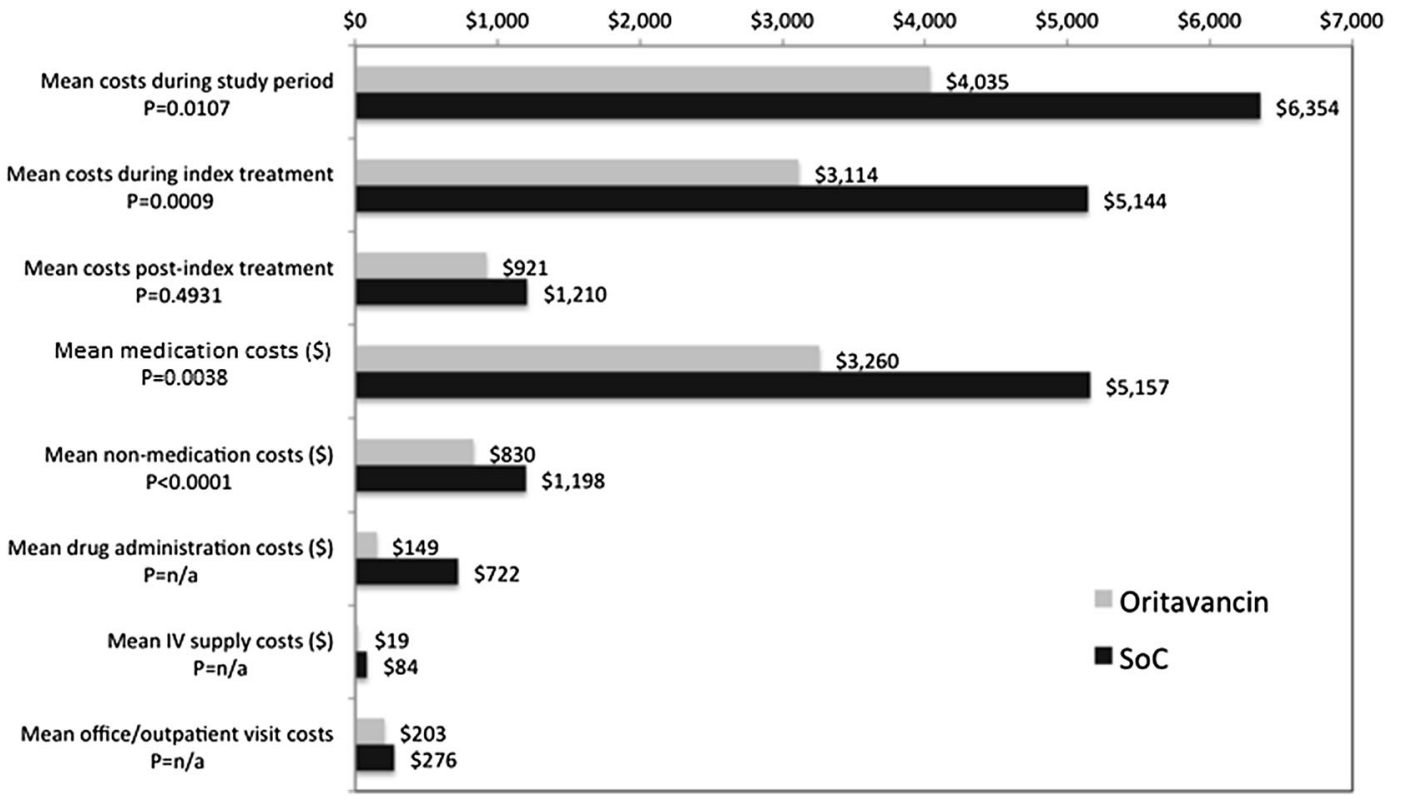

Fig. 2 Mean costs from initiation of study drug to end of study period

observed across all clinical subgroups, with patients diagnosed with wound infection demonstrating the greatest savings, at $\$ 5775$.

\section{Healthcare Resource Utilization}

Oritavancin patients required 1 day of therapy compared to a mean of 7.2 days of therapy for SoC patients. Approximately one-quarter of oritavancin patients $(n=16,27.1 \%)$ required additional post-treatment oral or IV antibiotics, but almost double this proportion of SoC patients $(n=31,52.5 \%)$ required additional antibiotics (Table 3 ).

In total, 26 patients had a hospital admission within 30 days prior to index treatment (11 oritavancin, 15 SoC). One SoC patient experienced an ER visit during the index treatment period for complications related to 


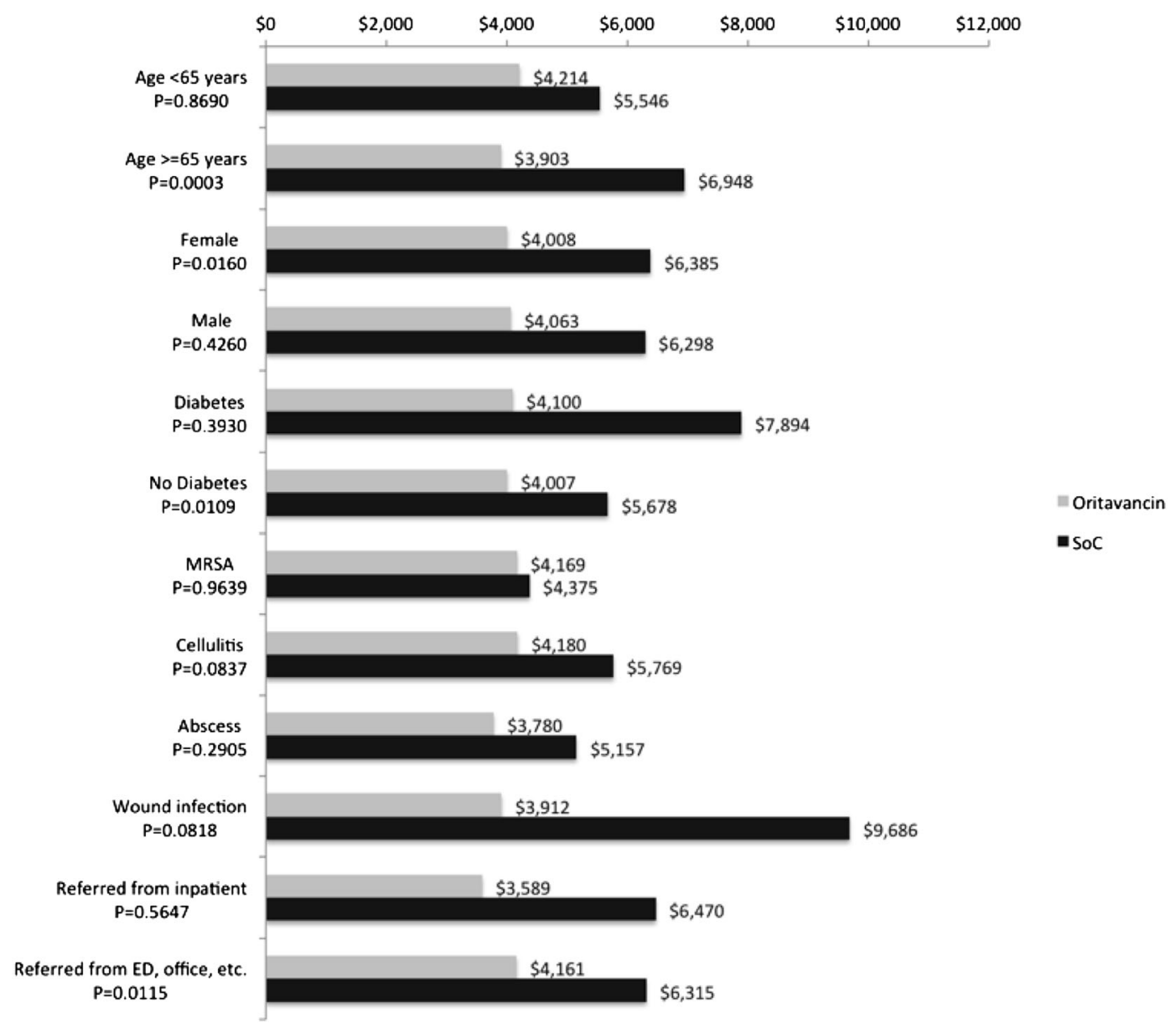

Fig. 3 Mean costs by patient subgroups

the peripherally inserted central catheter line. No oritavancin patients visited an ER during the index period. For the post-index treatment period, two patients in the SoC cohort had an ER visit and three patients in the SoC cohort were hospitalized. No patients in the oritavancin cohort were hospitalized or required an ER visit following index treatment (Table 3).

\section{DISCUSSION}

In the current healthcare environment, there is substantial pressure to reduce costs and resource use while maintaining or improving quality of care. This study analyzed the effectiveness of ABSSSI treatment in an outpatient infusion center with single-dose oritavancin compared to SoC antibiotics and found that oritavancin was clinically comparable to SoC antibiotics, but less costly and resource intensive.

For overall effectiveness (cured or improved), oritavancin showed similar rates $(90.2 \%$ cured or improved) to SoC antibiotics (77.4\%). Notably, the clinical cure rate was statistically higher for the oritavancin cohort (73.2 vs. $48.4 \% ; P=0.0315)$. The effectiveness rates in this real-world study were comparable to rates seen in clinical trials. The phase 3 SOLO1 trial compared single-dose oritavancin to 7-10 days of twice daily vancomycin. Oritavancin demonstrated comparable effectiveness for the primary composite endpoint (cessation of spreading or reduction in lesion size) at early clinical evaluation (ECE) at $48-72 \mathrm{~h}(82.3 \%$ for oritavancin, $78.9 \%$ for vancomycin, $95 \%$ CI $-1.6,8.4)$; investigator-assessed clinical cure at 
Table 3 Healthcare resource utilization

\begin{tabular}{lccc}
\hline & $\begin{array}{l}\text { Oritavancin } \\
(\boldsymbol{N}=\mathbf{5 9}), \boldsymbol{n}(\%)\end{array}$ & $\begin{array}{l}\text { SoC } \\
(\boldsymbol{N}=\mathbf{5 9}), \boldsymbol{n}(\%)\end{array}$ & $\begin{array}{l}\text { Total } \\
(\boldsymbol{N}=\mathbf{1 1 8}), \boldsymbol{n}(\%)\end{array}$ \\
\hline Patient visited ER & $12(20.3 \%)$ & $9(15.6 \%)$ & $21(17.8 \%)$ \\
Prior to index treatment & $0(0.0 \%)$ & $1(1.7 \%)$ & $1(0.8 \%)$ \\
During index treatment & $0(0.0 \%)$ & $2(3.4 \%)$ & $2(1.7 \%)$ \\
Post index treatment & & & \\
Patient hospitalized & $11(18.6)$ & $15(25.4 \%)$ & $26(22.0 \%)$ \\
Prior to index treatment & $0(0.0 \%)$ & $0(0.0 \%)$ & $0(0.0 \%)$ \\
During index treatment & $0(0.0 \%)$ & $3(5.1 \%)$ & $3(2.5 \%)$ \\
Post-index treatment & & & \\
Index ABX & $59(100 \%)$ & $0(0 \%)$ & $59(50.0 \%)$ \\
Oritavancin & $0(0 \%)$ & $3(5.1 \%)$ & $3(2.5 \%)$ \\
Vancomycin & $0(0 \%)$ & $15(25.4 \%)$ & $15(12.7 \%)$ \\
Dalbavancin & $0(0 \%)$ & $34(57.6 \%)$ & $34(28.8 \%)$ \\
Daptomycin & $0(0 \%)$ & $7(11.9 \%)$ & $7(5.9 \%)$ \\
Other IV ABX & $16(27.1 \%)$ & $31(52.5 \%)$ & $47(39.8 \%)$ \\
Patient received any non-study ABX post-index ABX, $n(\%)$ & $9(15.3 \%)$ & $18(30.5 \%)$ & $27(22.9 \%)$ \\
Received IV/IM ABX w or w/o oral & $7(11.9 \%)$ & $13(22.0 \%)$ & $20(16.9 \%)$ \\
Received oral ABX only & & & \\
\hline
\end{tabular}

$A B X$ antibiotics, $E R$ emergency room, $I M$ intramuscular, $I V$ intravenous, $S o C$ standard of care

post-therapy evaluation $(79.6 \%$ for oritavancin, $80.0 \%$ for vancomycin, $95 \% \mathrm{CI}-5.5,4.7)$; and lesion size reduction $\geq 20 \%$ at ECE $(86.9 \%$ for oritavancin, $82.9 \%$ for vancomycin, $95 \%$ CI $-0.5,8.6)$. [13] Similar results were seen in the phase 3 SOLO2 trial of single-dose oritavancin compared to twice daily vancomycin for 7-10 days. Oritavancin demonstrated comparable effectiveness for the primary composite endpoint at ECE (80.1 vs. $82.9 \%)$, investigator-assessed clinical cure at post-treatment evaluation (82.7 vs. $80.5 \%)$, and $\geq 20 \%$ lesion size reduction at ECE $(85.9$ vs. $85.3 \%$ ). Clinical response rates were similar across sub-groups, including BMI, age, MRSA, gender, and race [13].

This analysis also found that oritavancin demonstrated consistent clinical effectiveness across subgroup populations with various clinical characteristics, including MRSA. This consistency is supported by a recent analysis of pooled SOLO study results that used a modified Eron severity classification system for skin and soft tissue infections to evaluate oritavancin compared with vancomycin in patients with varying degrees of morbidity and disease severity. The study concluded that single-dose oritavancin was an effective alternative to multi-dose IV vancomycin for the treatment of ABSSSI patients within modified Eron Classes I-III and that management in the inpatient or outpatient setting had comparable efficacy [14].

Based on these study results, the clinical effectiveness of oritavancin and SoC IV antibiotics in the real-world outpatient setting is comparable to that seen in clinical trials. This study further found that while outcomes are comparable, outpatient treatment with 
oritavancin was less expensive and less resource intensive than outpatient treatment with SoC IV antibiotics, with a mean cost savings of $\$ 2319$ per patient. Cost savings were derived from both lower medication costs and fewer healthcare resources needed to deliver treatment. Importantly, this study also found that the cost of outpatient treatment with oritavancin was substantially lower than reported costs for inpatient management of ABSSSI. Ektare et al. estimated total costs to the payer of $\$ 9813$ to $\$ 18,014$ for inpatient treatment of ABSSSI [5]. A 2015 analysis by Lodise et al. based on a retrospective analysis of hospital discharge data from the Premier Research Database found that hospital costs for inpatient care ranged from $\$ 5851$ to $\$ 12,457$ for patients without life-threatening infections [3]. The Lodise analysis also determined that $94 \%$ of all hospitalized ABSSSI patients did not have a life-threatening infection, indicating that up to 535,100 hospital admissions in the US could be avoided with greater use of the outpatient setting $[2,3]$. Further, in this study the majority of patients had no prior hospitalization $(81.3 \%$ of oritavancin and $74.6 \%$ of SoC), and there was a low rate of ER visits and hospitalization following outpatient treatment ( $0 \%$ oritavancin vs. $3.4 \%$ SoC, NS; $0 \%$ oritavancin vs. $5.1 \%$ SoC, NS). This indicates that effective treatment can be provided entirely in the outpatient setting for most patients.

These findings and other recent investigations [8-11] provide a clear implication of the potential for cost savings in moving ABSSSI treatment from the inpatient to outpatient setting. When this is coupled with the cost savings obtained from using single-dose over SoC antibiotics, outpatient treatment of ABSSSI with oritavancin may provide much-needed cost savings. These cost savings accrue not only to the health care system, but also to patients, as many patients are responsible for a co-insurance of $\sim 20 \%$ on the cost of outpatient services. Further, oritavancin patients visited the infusion center only once for drug administration, compared to a mean of 7 visits for SoC patients. Given no difference in clinical outcomes, both the cost to the patient and overall treatment burden on patients should be considered when making appropriate treatment decisions.

\section{Strengths/Limitations}

A key strength of this analysis is that it includes both clinical outcomes and the economic impact of treatment with oritavancin in an outpatient center. Additionally, the results seen in the sensitivity analysis demonstrate the robustness of the primary effectiveness outcome, and sub-group analysis found that the results are consistent across multiple patient subgroups. Further, results are comparable to those seen in the two prior phase 3 clinical trials of oritavancin.

While this is the first study to look at oritavancin treatment for ABSSSI in an outpatient setting, several limitations should be considered when interpreting this study's findings. First, this was a retrospective, observational study performed at a single site. As such, it is subject to limitations such as lack of external validity, small sample size, and possible practice pattern differences such as type of SoC drugs used. Furthermore, while this study population is representative of ABSSSI patients typically treated at an outpatient infusion center site, it may not represent ABSSSI patients in other settings (such as hospitals, ER, hospital-based outpatient parenteral antibiotic therapy, or other outpatient centers). Further research is needed in other settings of care and with a larger sample size to validate the findings in this early study.

While this retrospective study captures real-world assessment and treatment choices, it is not known if this practice's choices are consistent with other institutions. Dalbavancin was used in $25 \%$ of the SoC group; however, Premier Hospital data show that national usage is less than 1\% of ABSSSI treatments in 2015 [15]. Notably, in this study only $20 \%$ of patients had lesion size recorded in the medical chart. While lesion size measurement is part of the US Food and Drug Administration definition of ABSSSI, it is not known how frequently lesion 
size is actually measured and recorded in the real world, although these study results indicate it is not an important criterion in treatment decision-making [16]. Additionally, while more than one-half of patients in this study had a follow-up visit that occurred within the assigned 5-30-day period, $30.5 \%$ of oritavancin and $47.5 \%$ of SoC patients did not have documented follow-up within this time frame; 2 patients had no outcomes data recorded; 5 patients had follow-up visits within the first 5 days; 13 patients had follow-up after the pre-specified 30-day window; 26 patients did not return to the infusion center for follow-up. This likely reflects the location of this infusion center in a popular vacation destination and with a large catchment area that reaches into the neighboring state, making it difficult for many patients to return for follow-up. Last, microbiology data were available for only $22 \%$ of patients, which may reflect the real-world practice of making treatment decisions without information on ABSSSI pathogen type.

\section{CONCLUSION}

This study, conducted in an outpatient infusion center, demonstrates that real-world treatment of ABSSSI with single-dose oritavancin provides equivalent outcomes compared with SoC antibiotics with 6 fewer days of treatment and a cost savings of greater than $\$ 2000$. It also provides evidence of lower costs relative to hospital inpatient care, an important consideration when hospital costs comprise one-third of total healthcare spending. In an era of continued pressure to reduce costs without compromising outcomes, outpatient treatment with single-dose oritavancin may present a solution to the management of ABSSSI patients.

\section{ACKNOWLEDGEMENTS}

The Medicines Company provided funding for this study and article processing charges. All authors had full access to all of the data in this study and take complete responsibility for the integrity of the data and accuracy of the data analysis.

The authors wish to thank Caitlin Rothermel of MedLitera for her assistance with editing and formatting this manuscript. The Medicines Company provided funding for this assistance.

All named authors meet the International Committee of Medical Journal Editors (ICMJE) criteria for authorship for this manuscript, take responsibility for the integrity of the work as a whole, and have given final approval for the version to be published.

Disclosures. Patrick J. Anastasio received funding from The Medicines Company for the conduct of this study. Peter Wolthoff received consulting fees from The Medicines Company. Annmarie Galli received consulting fees from The Medicines Company. Weihong Fan is an employee of The Medicines Company.

Compliance with Ethics Guidelines. All procedures followed were in accordance with ethical standards. A waiver of institutional review consent was granted by the New England Internal Review Board (NEIRB). This study was conducted retrospectively, and all protected, patient-identifiable information was not shared with the Sponsor or anyone outside of the study site. Data abstraction, de-identification, and entry were performed onsite at the infusion clinic by an independent Clinical Research Organization, Medical Data Analytics (MDA) of Parsippany, NJ.

All procedures followed were in accordance with the ethical standards of the responsible committee on human experimentation (institutional and national) and with the Helsinki Declaration of 1964, as revised in 2013. This article is based on previously completed treatment of patients and does not involve any new interventions performed on human or animal subjects by any of the authors. This was a retrospective study, informed consent was waived.

Open Access. This article is distributed under the terms of the Creative Commons 
Attribution-NonCommercial 4.0 International License (http://creativecommons.org/licenses/ by-nc/4.0/), which permits any noncommercial use, distribution, and reproduction in any medium, provided you give appropriate credit to the original author(s) and the source, provide a link to the Creative Commons license, and indicate if changes were made.

\section{REFERENCES}

1. Centers for Disease Control and Prevention (CDC). FastStats-Health Expenditures. http://www.cdc. gov/nchs/fastats/health-expenditures.htm. Accessed 30 July 2016.

2. HCUPnet. Healthcare Cost and Utilization Project (HCUP). Rockville: Agency for Healthcare Research and Quality. http://hcupnet.ahrq.gov/. Accessed 30 July 2016.

3. Lodise TP, Fan W, Sulham KA. Hospital admission patterns in adult patients with skin and soft tissue infections: identification of potentially avoidable hospital admissions through a retrospective database analysis. Hosp Pract. 2015;43(3):137-43.

4. Moran GJ, Krishnadasan A, Gorwitz RJ, et al. Methicillin-resistant $S$. aureus infections among patients in the emergency department. $\mathrm{N}$ Engl J Med. 2006;355(7):666-74.

5. Ektare V, Khachatryan A, Xue M, Dunne M, Johnson K, Stephens J. Assessing the economic value of avoiding hospital admissions by shifting the management of gram + acute bacterial skin and skin-structure infections to an outpatient care setting. J Med Econ. 2015;18(12):1092-101.

6. Hersh AL, Chambers HF, Maselli JH, Gonzales R. National trends in ambulatory visits and antibiotic prescribing for skin and soft-tissue infections. Arch Intern Med. 2008;168(14):1585-91.

7. Stevens DL, Bisno AL, Chambers HF, et al. Practice guidelines for the diagnosis and management of skin and soft tissue infections: 2014 update by the
Infectious Diseases Society of America. Clin Infect Dis. 2014;59(2):1-52.

8. Talan DA, Salhi BA, Moran GJ, et al. Factors associated with decision to hospitalize emergency department patients with skin and soft tissue infection. West J Emerg Med. 2015;16(1):89-97.

9. Tice A. Oritavancin: a new opportunity for outpatient therapy of serious infections. Clin Infect Dis. 2012;54(Suppl 3):S239-43.

10. Lane MA, Marschall J, Beekmann SE, et al. Outpatient parenteral antimicrobial therapy practices among adult infectious disease physicians. Infect Control Hosp Epidemiol. 2014;35(7):839-44.

11. Gilchrist M, Seaton RA. Outpatient parenteral antimicrobial therapy and antimicrobial stewardship: challenges and checklists. J Antimicrob Chemother. 2015;70(4):965-70.

12. David MZ, Daum RS. Community-associated methicillin-resistant Staphylococcus aureus: epidemiology and clinical consequences of an emerging epidemic. Clin Microbiol Rev. 2010;23(3):616-87.

13. Corey GR, Good S, Jiang $\mathrm{H}$, et al. Single-dose oritavancin versus 7-10 days of vancomycin in the treatment of gram-positive acute bacterial skin and skin structure infections: the SOLO II noninferiority study. Clin Infect Dis. 2015;60(2):254-62.

14. Deck DH, Jordan JM, Holland TL, et al. Single-dose oritavancin treatment of acute bacterial skin and skin structure infections: SOLO trial efficacy by Eron severity and management setting. Infect Dis Ther. 2016;5(3):353-61.

15. Premier, Inc. Premier Research Services. Charlotte; 2016. https://www.premierinc.com/transforminghealthcare/healthcare-performance-improvement/ premier-research-services/. Accessed Dec 12, 2016.

16. U.S. Food and Drug Administration, Center for Drug Evaluation and Research (CDER). Acute Bacterial Skin and Skin Structure Infections: Developing Drugs for Treatment. 2013. http:// www.fda.gov/downloads/Drugs/Guidances/ucm071 185.pdf. Accessed 30 July 2016. 